\title{
The Enhancement Effect at Local Reflectance and Emission Back to Apertureless SNOM Tips in the Shear-Force Gap
}

\author{
Gerd Kaupp*
}

\author{
University of Oldenburg, Faculty V, Diekweg 15, D-26188 Edewecht, Germany
}

\begin{abstract}
Physical basics, technique, equipment, proper experimental conditions, and performance of apertureless reflection back-to-the-fiber SNOM (Rayleigh, Raman, fluorescence) under shear-force control are reported. This technique achieves optical resolutions of $<10 \mathrm{~nm}$ on flat surfaces and $<20 \mathrm{~nm}$ in the presence of topographies with very sharp tips $(<10-20 \mathrm{~nm}$ end radius). It works due to the large enhancement of the reflection to very sharp tapered uncoated tips in the shear-force gap. The dependence of preset vibration damping is linear with variations between different chemical entities. Thus, the optical contrast appears as chemical contrast in reflection or emission. Such enhancement is however not obtained with blunt tips (>30 nm end radius or broken). Typical images for all thinkable situations show that topographical artifacts are excluded and that, unlike conventional aperture SNOM, precise site coincidence of optical contrast and chemical variation (often traceable in the topography) is obtained on planes, hills, slopes, depressions, and hidden geodes, even on very rough surfaces that give, however, no optical contrast if they are chemically uniform. Furthermore, positive and negative contrast on hills, slopes, and depressions is featured within the same SNOM image each. Object sizes range from $30 \mathrm{~nm}$ in small scans to $25 \mu \mathrm{m}$ in $40 \mu \mathrm{m}$ wide scans. The versatile technique is therefore qualified for applications on real world samples without severe manipulations just as they occur in science, technique, and medicine. The principal differences of apertureless SNOM with collection in the shear-force gap and conventional SNOM are worked out.
\end{abstract}

Keywords: Apertureless SNOM, chemical contrast, local spectroscopy SNOM, optical resolution at $10 \mathrm{~nm}$, reflection enhancement, rough surfaces, sharp dielectric tip, shear-force gap.

\section{INTRODUCTION}

The applications of apertureless scanning nearfield optical microscopy (SNOM or NSOM) with optical shear-force control have already been reviewed $[1,2]$. By virtue of the reflectance enhancement in the shear-force gap the easy technique could be used on surfaces with low to high corrugation. Studies on nanoparticles (size, aggregation, performance), biological objects, cell organelles (details), (pre)cancer diagnosis, blood bags' performance, dental alloys' corrosion, solid-state chemistry, petrified bacteria, natural geodes, glazed paper, local Raman spectroscopy, local surface enhanced Raman spectroscopy (SERS), local fluorescence spectroscopy, detection of diffusion coefficients, and assessment of light fastness of dyed textile fibers have been covered. Countless further applications in materials science are equally possible. Unexpected new insights are obtained in all of the various fields that cannot be obtained by any other technique. Nevertheless, this robust and versatile technique did not find inclusion in recent reviews on SNOM that are still restricted to very blunt metal-coated aperture tips (the widths are $50-150 \mathrm{~nm}$ plus twice the thickness of the metal coating of $100-200 \mathrm{~nm}$ ) with low light transmission, extreme tip-sample convolution, topographical artifacts, high temperature when used as nanoscopic light sources, and inherently low submicroscopic resolution [3-6].

\footnotetext{
*Address correspondence to this author at the University of Oldenburg, Faculty V, Diekweg 15, D-26188 Edewecht, Germany; Tel: +49 4486 8386; Fax:+49 4486 920704; E-mail: gerd.kaupp@uni-oldenburg.de
}

This is rather strange, because reflection back-to-theuncoated-tip in the shear-force gap technique provides the genuine optical contrast precisely at the site where it occurs, whereas all techniques with metal-coated aperture tips must show the optical contrast at uncontrolled sites well away from the source for its occurrence, which detracts such SNOM from practical applications. The reasons for the apparent non-awareness might be the necessity of well defined although easily executed experimentation, and failure if these are not acknowledged [7]. Our technique requires sharp uncoated tips with high aspect ratio and rapid optical shear-force control to avoid tip breakage in fast scans. SNOM tips will however inevitably break if etched silica fibers (too blunt with large taper angles of $30^{\circ}-50^{\circ}$ ) are used to scan on platinum (a material with exceptionally poor shear-force response) so that only artifacts arose that were rightfully withdrawn in [7] because of the undue experimentation. Importantly, the proper experimentation requires an intensity meter for uninterrupted check of the tip quality. The easily pulled tip (at almost no cost) must not be abraded or broken and that is most easily controlled with the intensity meter. Ease of operation and the always secured physical effect of strong enhancement of the reflected light in the shear-force gap back-to-the-sharp-tip enabled valid SNOM in virtually all branches of science and daily life with excellent repeatable interpretable contrast and without topographical artifacts $[1,2]$. This deserves a timely review on the basics of our apparently widely unexpected effect that deals with near-field behavior of materials when illuminated in the shear-force gap [8]. 


\section{TECHNIQUES AND EXPERIMENTS}

Essentially three ways for subwavelength resolution with light exist, which is SNOM (or NSOM), stimulated emission depletion (STED) using focal and doughnut-focal beam shapes, and microscopy with superlenses out of negative index of refraction materials (NIM). In the NIM technique objects are imaged with a resolution far finer than the illuminating wavelength, because NIMs, unlike conventional materials, make light to refract inward rather than outward, due to the negative index of refraction [9]. Flat nanolenses of this kind can be used as light couplers in nanooptics [10]. This does not describe typical behavior of the surface material, but rather particular superlens effects. STED uses confocal femto- and picosecond two-laser scanning with a claimed axial resolution of 33-46 nm of fluorescence spots $[11,12]$. It is limited by orientation requirements not allowing for considerable roughness. Furthermore, it is restricted to fluorescent objects or to materials that are stained with fluorescent dyes. This provides problems with photobleaching in the intensely illuminated laser fields.

Most SNOM techniques are comparatively complicated and lack the capability for scanning rough surfaces. They shall be briefly characterized for working out the principal differences with the apertureless shear-force technique that is the subject of this review. SNOM techniques are manifold but all of them use confined light in some way. Fig. (1) lists the various possibilities. They subdivide in apertured (metalcoated) fiber tips, transparent or opaque atomic force microscopy (AFM) tips, and apertureless (uncoated) fiber tips. Fig. (2) shows the principal differences of apertured and apertureless (uncoated) tips. Metal-coated tips (a) cannot follow rough surfaces in near constant distances. If for example the right edge of the tip (a) would ascend the right slope at about $5 \mathrm{~nm}$ shear-force distance the center of the aperture would be about $500 \mathrm{~nm}$ above the bottom of the depression, so that topographical artifacts in the optical response were inevitable. Even when staying in the nearfield $(<\lambda / 2)$ the optical response would sensibly depend on the distance. If the metal-coated tip would be applied with inclination (for example at $20^{\circ}$ from perpendicular) mostly the deepest edge point of the coating would in "tapping mode" lift up the aperture by a distance of $\sin 20(m+0.5 d)$ $\mathrm{nm}$, where $m$ is the depth of the metal coating and $d$ the diameter of the aperture. This would decrease the tip surface convolution in the AFM at the expense of additional complications with artifacts in the SNOM. Severe topographical artifacts by distance variations are also occurring if the tip (a) is scanned at a constant height above the highest peak of the surface.

Fortunately, uncoated sharp tips with high aspect ratio (Fig. (2b, c) can easily follow such topography under proper shear-force control without producing topographical errors in AFM and reflection enhanced SNOM. An interesting modification of technique D in Fig. (1) with sharp particle between support and aperture at AFM distance control has recently provided collection enhancement $[13,14]$. This is very similar to reflection enhancement; the illuminated sharp protrusions are imaging the extended coating and aperture.

Despite the difficulties with extremely blunt apertured tips that cause high costs and low light throughputs with strong up-heating when used as light sources, these are still more popular than the sharp apertureless tips $(b, c)$ that profit from the widely unexpected reflectance enhancement effect in the shear-force gap and do not have similar technical problems and do not noticeably charge the budget when pulled with a commercial pipette puller.
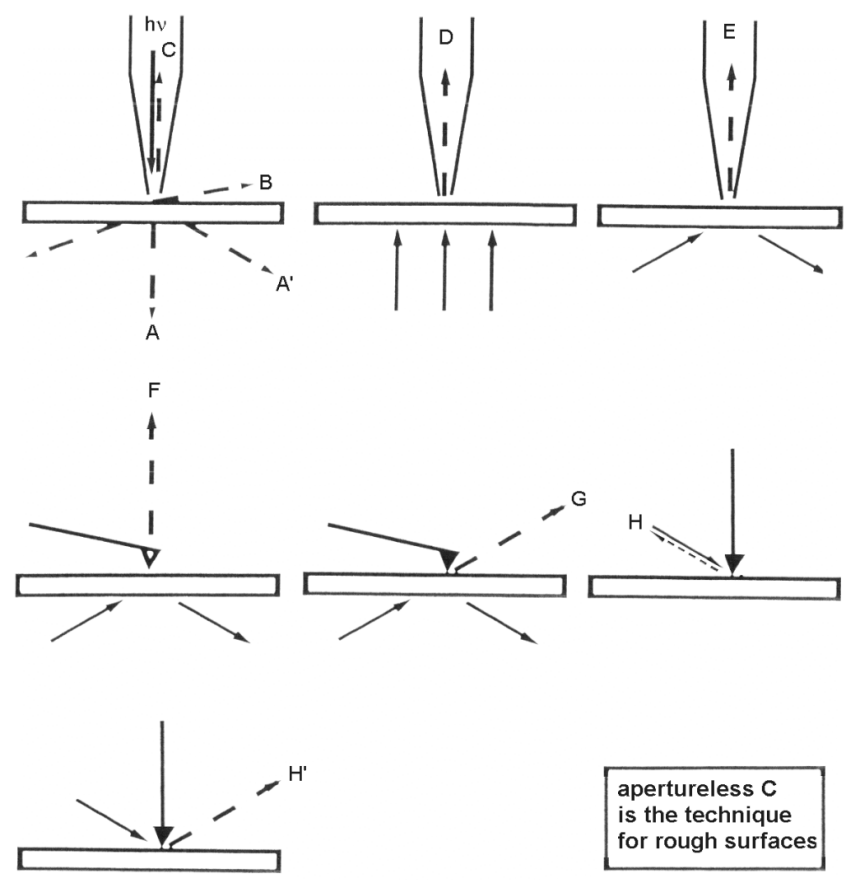

apertureless C

is the technique

for rough surfaces

Fig. (1). The various principles of SNOM techniques; aperture SNOM (A, A', B, C) with metal coated light sources or collectors $(\mathrm{D}, \mathrm{E})$; apertureless SNOM by light tunneling $(\mathrm{F}, \mathrm{G})$; apertureless scattering at a near- field contact $\left(\mathrm{H}, \mathrm{H}^{\prime}\right)$; apertureless version of geometry $\mathrm{C}$; solid arrows illumination; broken arrows collection.
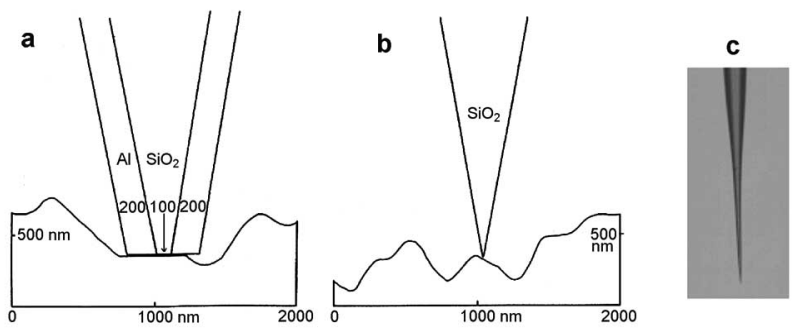

Fig. (2). On scale sketch of (a) a typical metal-coated tip, (b) a sharp uncoated tip, and (c) photo of an uncoated tapered tip indicating the high aspect ratio; the tips in (a) and (b) are placed in the shear-force distance of about $5 \mathrm{~nm}$ [2].

A brief description of the experimentation with conventional SNOM and the more recent highly involved apertureless techniques is therefore in order for comparison with the present technique. AFM tips use contact or tapping mode control, whereas apertured and apertureless tips are mostly horizontally vibrated for shear-force damping control. But metal-coated tips can also be used in tapping mode in particular for measurements of life objects in water when the tip quality factor (Q-factor) was improved. However, $500 \mathrm{~nm}$ wide metal-coated tips usually abrade the surface roughness and the lateral optical resolutions are not better than about $100 \mathrm{~nm}$ [15]. The techniques A, A' and B in Fig. (1) collect in the far-field and the angles depend on 
the index of refraction. $\mathrm{C}$ and $\mathrm{D}$ will not experience the enhancement that is restricted to very sharp tips. The techniques A' and E collect forbidden light, $\mathrm{E}$ with near-field collection. The improved photon scanning tunneling microscopy (PSTM) techniques $\mathrm{F}$ and $\mathrm{G}$ belong to apertureless SNOM. They rely on collection of forbidden light that is illuminated through the sample at angles of total reflection by a sharp transparent $(F)$ or opaque tip $(G)$ on the flat surface [16]. This is genuine near-field enhancing of the light intensity above zero. But it is not often applied due to experimental difficulties with interference between the tip field and the background field, and the fringes may obscure the SNOM image [17]. Furthermore, even very minor topography and local changes of the refractive index combine to the optical signal. Separations have been tried by a distance modulation technique [18]. Technique $\mathrm{H}$ is scanning interferometric apertureless microscopy (SIAM). It uses double modulation with reflection of the light from an interferometer back to the interferometer and records the phase shift due to scattering at an AFM tip at the other side of a transparent plate with reference to a second spot without such scattering. Resolutions down to $1 \mathrm{~nm}$ have been claimed [19], however the origin of the high-resolution features of a microscope cover-slip remained unexplored and the image is recognizably artificial [2]. The technique H' focuses laser beams to the apex of metal tips as scattering devices that are in tapping mode to the surface, in order to obtain field enhancement under the metal tip and demodulation of the signal at higher harmonics [20]. Clearly, this technique is again restricted to very flat surfaces and it remains to be shown whether materials' interfaces would be scanned with high contrasts by these techniques. More detailed descriptions of the theory, various experimental setups, and artifacts of scattering apertureless SNOM (H and $\left.\mathrm{H}^{\prime}\right)$ are given in [21].

Fortunately, the robust SNOM technique $\mathrm{C}$ but for uncoated tips (cf. Fig. 1) is less complicated, provides optical resolution equal to AFM resolution (or better), and avoids problems even with highly rough surfaces. It uses reflection back-to-the-uncoated-sharp-tip and was the first technique of apertureless SNOM [2, 22-24]. These tips are used both as emitter and enhanced reflection acceptor in the shear-force gap with undisturbed fast optical shear-force damping control. The standard rule for aperture SNOM that topography and optical contrast must not coincide is therefore replaced by the favorable "golden rule for apertureless shear-force SNOM": The optical contrast must be found precisely at the site where it is generated for being true. If it is displaced, the output was artificial because of unsuitable experimentation. We are comparing totally different techniques that depend on different physical effects. It is therefore timely to remove severe misunderstandings and prejudices, inasmuch as our technique is outstanding for all of the important applications of SNOM, which are listed in the Introduction. There is no cross-talk between our SNOM and AFM, because we profit from the enhancement effect [1,2, 22-24], and if there is remaining doubt one can use a filter for removal of any presumed light from the control laser. This will be exhaustively demonstrated below. For example, we present both positive and negative optical contrast of different particles all in one repeatable image (cf. Fig. 14), and our contrasts are interpretable. The present situation enforces a detailed description of the experimentation and physical basis of apertureless shear-force SNOM by enhanced reflection back-to-the-sharp-illuminating-tip.

\section{EQUIPMENT FOR SNOM ON ROUGH SURFACES WITHOUT TOPOGRAPHICAL ARTIFACTS}

The block diagram of the commercial instrument (RASTERSCOPE SNOM 4000, Danish Micro Engineering $\mathrm{A} / \mathrm{S}$, Copenhagen) is depicted in Fig. (3) $[2,25]$. A photograph indicating the easy instrument mount is available in [2]. The crossed polarizers at (1) remove straylight of the operating laser $(488.0 \mathrm{~nm}$ ) (e.g. model 161 LGS from Laser Graphics or stable laser with other wavelength). The light is coupled to a mono- or better multi-mode fused coaxial quartz waveguide with core diameter of 3.4 or $5.7 \mu \mathrm{m}$ and $125 \mu \mathrm{m}$ step index at lengths of $60-120 \mathrm{~cm}$ that loose all of the original polarization. The fiber (quality- or Q-factor is about 100) is pulled to $<10-20 \mathrm{~nm}$ end radius with a laser puller such as the P-2000 micropipette puller (Sutter Instruments, Navato, CA).

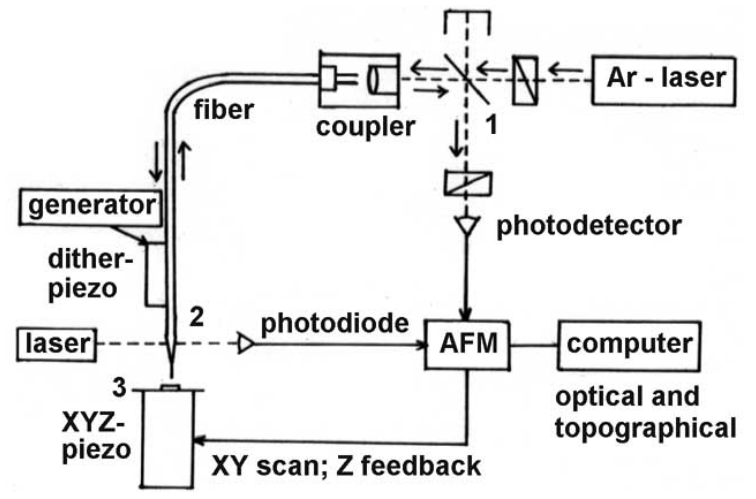

Fig. (3). Block diagram of an apertureless reflection back-to-thefiber SNOM instrument with shear-force control and crosspolarization only to minimize straylight; (1) beam splitter and crossed polarizers; (2) shear-force arrangement; (3) sample mount on a piezo stage [2].

The depolarized (checked with a polarizer) light intensity at the end of the tip on an about $1 \mu \mathrm{m}$ wide spot may reach $60-80 \mu \mathrm{W}$. The system operates with optical shear-force control (2) $[26,27]$. The tip vibrations are in the range of $100-400 \mathrm{kHz}$ with free amplitudes of 5 or $10 \mathrm{~nm}$ and the damping settings are fixed between $30 \%$ and $60 \%$ depending on the materials. Very fast feedback response is obtained when the vibrating tip shadows a $\mathrm{He} / \mathrm{Ne}$ laser $(632.8 \mathrm{~nm})$ beam. The AFM and simultaneous SNOM scanrates are $2-120 \mu \mathrm{m} \mathrm{s}^{-1}$ at $512 \times 512$ pixels or $200 \mu \mathrm{m} \mathrm{s}^{-1}$ for larger scans (mostly $2 \mathrm{~min} /$ frame). Acoustic control by tuning fork or mechanical control by bimorph, as glued to fiber tip, was too slow, requiring detrimentally slow scanning rates. The light reflected back to the fiber in the constant shear-force gap couples out and passes the beam splitter at (1) (the analyzer also halves the intensity) to the photomultiplier for the optical image. An energy meter (alternatively an XY plotter) (e.g. Hamamatsu, model H5783-01) is connected for the control of proper experimental conditions. Appropriate filters can be placed in front of the photodetector if the operating laser light shall be removed from emitted light, but control-laser light was not 
detectable at this point. Alternatively, the light can be diffracted by an optoacoustic spectrometer with waveguide optics. Approach to the shear-force damping position is in two steps: coarse and cautiously close. The fast optical feedback dynamics was checked with consecutive AFM scans on porous silicon samples (50 peaks and depressions in an area of $121 \mu \mathrm{m}^{2}$; nominal depth $2 \mu \mathrm{m}$, diameter $1.5 \mu \mathrm{m}$ ) at $70 \mu \mathrm{m} \mathrm{s}^{-1}$, when numerous height variations of $2.5 \mu \mathrm{m}$ were recorded [2]. Calibrations for width and height used an interferometric fabricated three-dimensional (3D) gold double grating (200 $\mathrm{nm}$ gold layer on silicon), type CAL 3000/500-A from IMS, Roskilde.

\section{PHYSICAL BASIS OF ENHANCED REFLECTANCE IN THE SHEAR-FORCE GAP}

The illuminated spot under the uncoated tip is about 1 $\mu \mathrm{m}$ in diameter. It might therefore appear strange that highly resolved SNOM should be obtainable with these. It was Courjon et al. [28] who proposed the possibility for apertureless SNOM with uncoated illuminated tips and reflection back to the fiber. They tried to use a phase effect for the reflection at near-field distance $(<\lambda / 2)$ but such effect could not be usefully verified. But we thought of near-field enhancement of reflected light and realized that the setup of Fig. (3) provided unexpectedly high reflection enhancements upon proper experimentation with sharp uncoated tips. These enabled to obtain genuine SNOM images since 1995 [29]. We further observed that blunt or broken tips did not provide the necessary enhancement but only artificial contrast in the optical channel. The reason for our success was further documented by recording the light intensity changes upon approach to the surface with an XY plotter as in Fig. (4) [30].

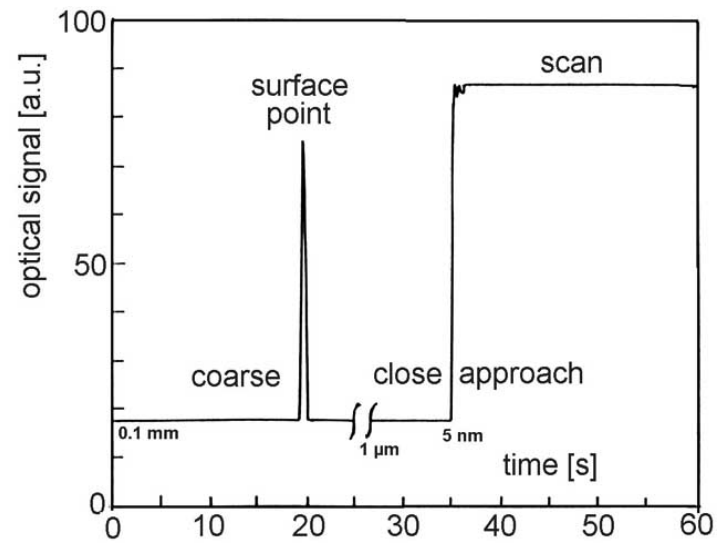

Fig. (4). Approach of an illuminated sharp multimode tapered quartz-glass tip to a uniform surface while monitoring the signal level, showing the strongly enhanced light intensity only while the tip vibration was being damped in shear-force distance $[8,30]$.

The fast coarse approach of the laterally vibrating tip is triggered by the damping control to stop, retract the tip by $1 \mu \mathrm{m}$ above the surface point, and hold it there to prevent penetration of the tip into the surface. Slow close approach is started from that waiting position until the predefined damping of the vibration is reached and scanning of the surface starts. Clearly, the basic far-field light level remains independent of the tip surface distance until the vibration damping is reached at the surface point when a short flash of near-field light is recorded. The original far-field intensity is reinstalled in the waiting position. Upon start of smooth close approach (right part of Fig. 4) the constant far-field signal intensity remains constant until the tip reaches the shear-force gap at the preset damping when again the large increase of the optical signal is obtained and the scanning of SNOM under shear-force distance control is started at the enhanced reflection level; the control provides AFM. Differences of the enhancement efficiency are recorded at the enhanced level. This works with different efficiency for all types of materials. Clearly, our unexpected enhanced reflectance when the sharp vibrating dielectric tip is in the shear-force gap (about $5 \mathrm{~nm}$ distance is generally accepted) deals with optical near-field reflectivity properties of materials. The scanning of variations in the enhanced nearfield reflection provides genuine SNOM with optical resolution below the tip radius. It distinguishes different materials but not topography. The constant far field light (Fig. 4) is only additive background that does not contribute to the optical contrast. As our effect relies on materials' properties in the shear-force gap, it has nothing in common with depolarization SNOM using uncoated tips at constant height (instead of constant distance) with a polarization manipulator [31].

The sharpness requirements for obtaining the enhancement of reflectance (also of fluorescence and Raman emission) are severe. The apex radius of the silica fiber must be $<20 \mathrm{~nm}$ or better $<10 \mathrm{~nm}$. Blunt $(>30 \mathrm{~nm}$ ) or broken tips (unless these exhibit a very sharp protrusion [2]) do not give reasonable enhancement but may even decrease the signal level while in shear-force distance. This is clearly shown by the approach curve of a broken tip in Fig. (5). Here the intensity decreases below the far-field background at the surface point and during undue shear-force scanning. In the absence of enhanced light no SNOM signal is obtained but far-field artifacts as with other SNOM types.

Every single measurement must control the signal level, as the near field enhancement is absolutely necessary. If the signal level drops considerably the tip has broken and the SNOM measurement should be discontinued. When too strong damping settings abrade the tip the enhancement is reduced. Blunt tips (e.g. etched tips) rapidly break at shearforce distance.

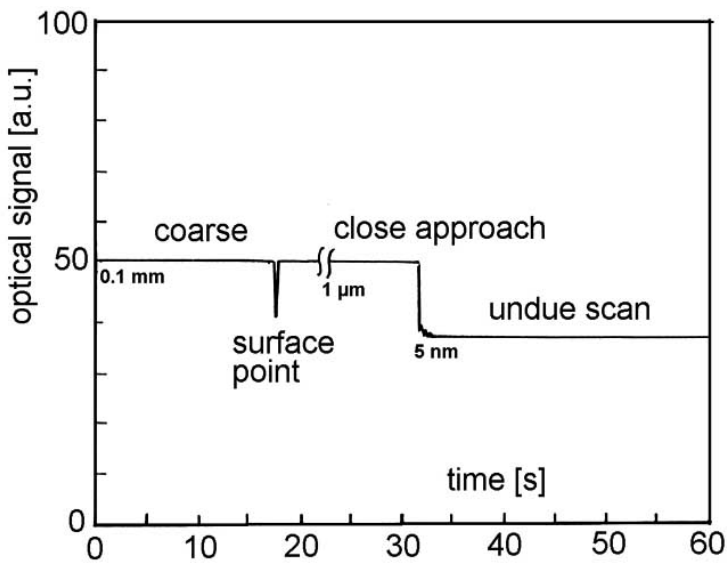

Fig. (5). Approach of a broken multimode tapered quartz-glass tip to a typical surface while monitoring the signal level; only artificial far-field artifacts are obtained under such unsuitable conditions of attenuated intensity $[8,30]$. 
An interesting modification of technique D in Fig. (1) with sharp particles between support and metal-coated tip (200 nm aperture) at contact or tapping mode AFM control was recently used for light localization and focusing. Illuminated $\mathrm{SnO}_{2}$ bumps with mean lateral dimensions of $550 \mathrm{~nm}$ and heights of $700 \mathrm{~nm}$ provided illuminated protrusions $[13,14]$. Such collection enhancement is very similar to the present reflection enhancement. Approaches of a $600 \mathrm{~nm}$ wide tip to the spiky illuminated $\mathrm{SnO}_{2}$ sample hits only the highest protrusions that image the rather flat surface. When the protrusions reach the aperture region the light collection by the tip enhances at AFM distance and that has the same or similar (other light path and AFM gap) origin as the signal enhancement in Fig. (4). This important new result will add to the physical understanding of the effect that is analyzed in the next section. It will be interesting to know if the stringent sharpness requirement in shear-force distance enhancement can be lowered by the tip imaging experiment with AFM distance.

\section{PERFORMANCE OF THE REFLECTANCE ENHAN- CEMENT IN THE SHEAR-FORCE REGION}

Enhancement factors are with respect to the residual farfield light that should be kept as low as possible with an additional iris stop $[2,8]$ if comparisons are made between materials. An enhancement factor $F=I / I_{0}$ has been defined where $I$ is the total intensity and $I_{0}$ the far-field background intensity (cf. Fig. 4). Factors of $F \geq 2$ are sufficient for pure optical contrast with poorly near-field reflecting compounds, but they may reach values of up to $\geq 50$ (e.g. on an ambient Al test sample). Optimal damping settings are not too low (sensitivity) and not too high (tip abrasion). It is therefore not only for physical but also for practical reasons important to know how the enhancement effect depends on the distance within the shear-force gap and how that changes with different materials. It is to be expected that the preset damping efficiency be closely related to the achieved shearforce distance. Such relation is revealed by approach curves that plot signal level or enhancement factor $F$ against various preset damping efficiencies under otherwise constant conditions. Figs. $(6,7)$ give typical examples $[2,8]$.

All points of a curve have been obtained with the same vibrating tip by successively increasing the preset damping value, surpassing the final decline of the efficiency. The tips were abraded beyond the decline point with a risk of being broken. Scanning electron microscopy (SEM) secured sharpness retention of tips that were only used in the linear part and blunting to those that experienced the horizontal or descending part. Clearly, the optimum for the safe SNOM measurement is to be sought in the middle of the linear part of the curves in Fig. (6) or (7), which depends on the materials' specific damping response and on the tip radius. More vibration damping that means shorter distance gives more enhancements with the unaffected tip. It is of high interest that the increase of the signal or of the factor $F$ is linearly related with the damping value. The sensitivity of the process is obtained from the slope $\Delta F /(\Delta \%$ damping $)$ of the linear part of the curves. These and the slopes of some other materials are collected in Table $\mathbf{1}$.
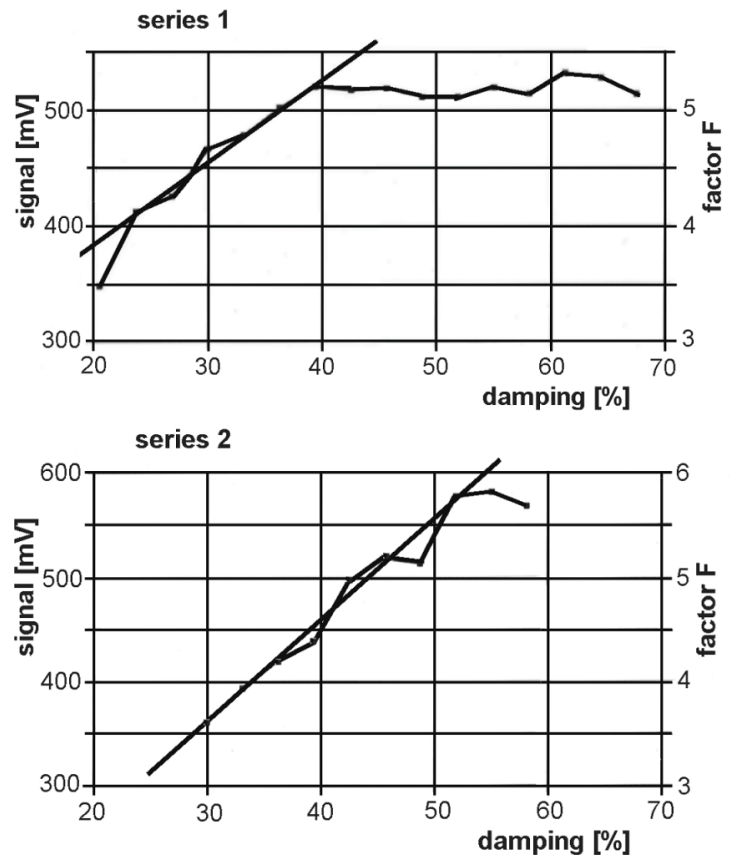

Fig. (6). Two different approach curves to a gold surface that had previously been treated with $\mathrm{H}_{2} \mathrm{~S}$, showing linear parts in the optimal damping regions without tip abrasion, followed by abrasion at too high damping; different tips and humidity were used in series 1 and $2[8]$.

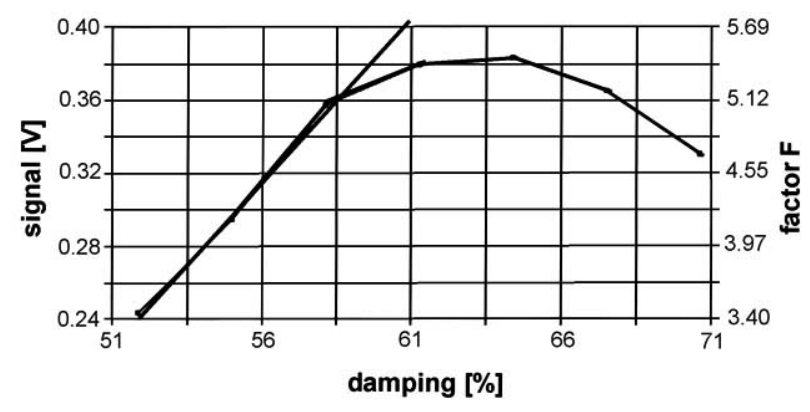

Fig. (7). Approach curve to a polished dolomite $\left(\mathrm{CaCO}_{3} \cdot \mathrm{MgCO}_{3}\right)$ surface, showing linear dependence prior to continuous tip abrasion in the bent part of the curve at higher damping settings [8].

All measurements in Table 1 were performed up to the abrasion or breakage of the tip. Despite uncertainties with the initial tip radii for the different curves, the values suffice for rough comparisons of the enhancement sensitivity in the optimal shear-force gap range [8]. They indicate the generality of the effect with very different types of materials and shear-force mechanisms. The slope values cover two magnitudes and that is the reason for the exceptionally strong optical contrast that is achieved with this type of apertureless SNOM even at small chemical differences [1, $22,23,30]$. Similar slope values were obtained when using $632.8 \mathrm{~nm}$ light [8]. Thus, wavelength dependences are at least minor in the visible region. However, the measured slopes are dependent on the ambient relative humidity, which was about twice as high in summer than in winter, both at room temperature (Table 1). Clearly, the specific 
Table 1. Steepness of the Approach Curve of Different Materials with one Silicon Tip Each, Exhibiting Average End Radius of 15 $\mathrm{nm}$, at $488 \mathrm{~nm}[2,8]$

\begin{tabular}{|c|c|c|}
\hline Material Surface & $\Delta \boldsymbol{F} /(\boldsymbol{\Delta} \%$ Damping $)$ & Type of Material \\
\hline \hline anthracene $(001)$ & 0.0064 & organic, unpolar \\
\hline phthalimide with water layer & 0.096 & organic, polar \\
\hline aluminum $+\mathrm{Al}_{2} \mathrm{O}_{3}$-layer and $\mathrm{H}_{2} \mathrm{O}$ & 0.025 (winter) & metal with hydrated oxide \\
\hline aluminum $+\mathrm{Al}_{2} \mathrm{O}_{3}$-layer and $\mathrm{H}_{2} \mathrm{O}$ & 0.040 (summer) & half-metal with hydrated oxide \\
\hline silicon + silica-layer and $\mathrm{H}_{2} \mathrm{O}$ & 0.373 & precious metal with S-H monolayer \\
\hline gold-S-H, series 1 & 0.069 (winter) & precious metal with S-H monolayer \\
\hline gold-S-H, series 2 & 0.099 (summer) & salt with water layer \\
\hline dolomite with water layer & 0.265 & \\
\hline
\end{tabular}

thickness of water layers is part of the effect and an important factor for the SNOM contrast. The most frequent and highly efficient shear-force mechanism seems to rely on the water layer at the tip and at the sample. Humidity influences on the shear-force have been reported [32]. We conclude that thicker water layers increase the sensitivity of the reflection enhancement. The water layers of quartz-glass tip and sample will certainly merge at about $5 \mathrm{~nm}$ distances. Also the thiol groups on the gold surface can accept a water layer. The lowest sensitivity in Table $\mathbf{1}$ was found for the hydrophobic hydrocarbon anthracene without water layer. In this case the shear-force distance must be smaller than in the other materials at the same damping settings, but water contact will be enforced from the layer on the tip upon close approach. Also platinum has no water layer and it is difficult to scan in shear-force mode with uncoated tips [8]. The most reasonable merging of the water layers apparently serves the coupling of the light in the shear-force gap. Therefore, inclusion of the merging concept should be considered for any modeling or simulation of the experimentally secured enhancement effect $[1,8,24,30]$. Unfortunately, the shearforce is still not fully understood [27] due to numerous factors and very diverse situations that are also reflected by the strong chemical contrasts in apertureless shear-force SNOM. Variations in the damping efficiency are clearly part of the optical contrast, as different distances are installed for different materials. Similar measurements of other SNOM techniques do not exist. The apertureless SNOM contrast differentiates materials with important practical applications.

\section{OPTICAL RESOLUTION}

A clear indication of the high lateral resolution power in the shear-force gap by enhanced reflection-to-the-sharpuncoated-tip was obtained from fluorescing nanoparticles in a resin, partly emerging over the surface. The single covered particles exhibited widths of $110-210 \mathrm{~nm}$ in the AFM and consistently $100-200 \mathrm{~nm}$ in the SNOM image. The nominal diameters were 100-200 $\mathrm{nm}$. The depth of the resin cover on the correctly measured nanoparticles could therefore be judged to be $5 \mathrm{~nm}$ and the optical resolution is better than the AFM resolution [33]. In another experiment a skew (slope of $10^{\circ}-15^{\circ}$ ) anthracene surface with anthraquinone features on it exhibited several $18-20 \mathrm{~nm}$ peak-to-peak distances in the optical image [23]. Another resolution criterion is the horizontal distance top-to-bottom at the contrast edge. Fig. (8) contains optical resolution values of 18-20 nm on a polycarbonate test sample with gold projections [8]. Also stripes artifacts at vertical surfaces, for example of cubic $\mathrm{NaCl}$ crystals of about $1 \mu \mathrm{m}$ height were resolved top-tobottom with $17.6 \mathrm{~nm}$ [8]. Similarly, the stripes artifact at the overhangs in Fig. (20) below are resolved to better than 20 $\mathrm{nm}$. Madsen et al. reported a similar lateral resolution (15 $\mathrm{nm}$ ) [34]. The best optical resolution is however obtained with contrasts at flat sites. Corresponding test samples were fabricated with minerals containing geodes. These were polished until about half of a geode reached the surface for a vertical interface (Fig. 9) [2, 8]. For example, the interface on marble in Fig. (10) gave a lateral top-to-bottom optical resolution of $8.6 \mathrm{~nm}$. This excellent resolution (better than $10 \mathrm{~nm}$ ) is inherent in the apertureless SNOM and very important for the optical imaging of nanoparticles, cell organelles, and the like even at high topographies.

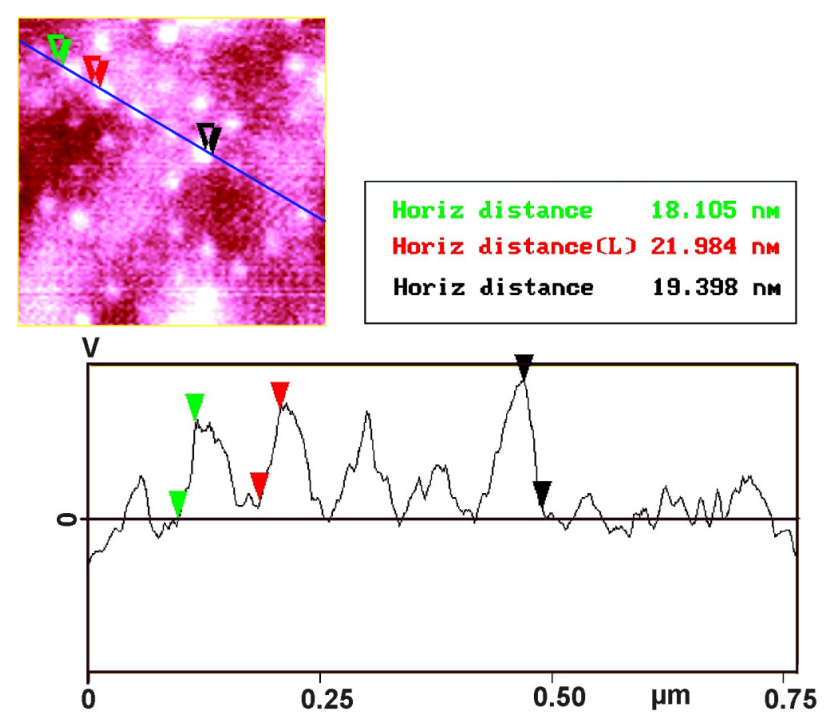

Fig. (8). Apertureless shear-force SNOM (632.8 nm light); top-tobottom resolution of triangular gold projections on a polycarbonate test sample; height of gold: $15 \mathrm{~nm}$ [2]. 


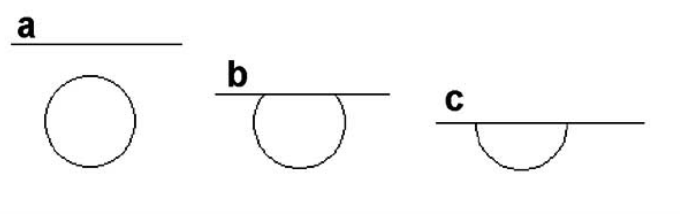

Fig. (9). Sketch for the use of geodes for test samples on flat surfaces by creating vertical interfaces; (c) is the best test sample [2].
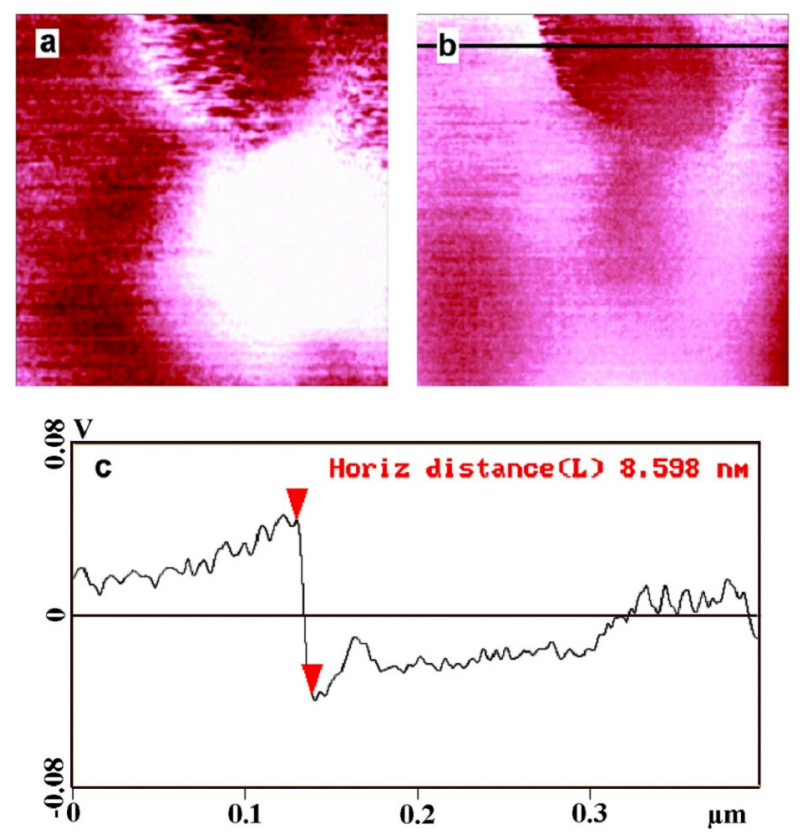

Fig. (10). Apertureless shear-force AFM (a) (z-range: $50 \mathrm{~nm}$ ) and SNOM (b) with a polished marble surface; optical resolution (c) at a geode interface $[2,8]$.

\section{CHEMICAL CONTRAST BY ENHANCED REF- LECTION AND EMISSION IN THE SHEAR-FORCE GAP}

Genuine SNOM provides contrast that differentiates chemically different materials (including different polymorphs). Thus, optical images of chemically uniform gold gratings are not chemical contrast but rely on far-field light concentration by mirror effects [22]. True chemical contrast $[22,23]$ must occur precisely at the site of the chemical species for being credible (the "golden rule"). This is also the basis for local spectroscopy if enhanced fluorescence or Raman emission is detected. Any nonchemical contrasts (that occur beyond geometrical limits at very sharp edges or almost vertical slopes and overhangs) are artificial even if they occur next to genuine chemical contrast in the same image. Chemical contrast may be caused on hills, depressions, slopes, and flat parts, wherever a change of materials resides. The sign of the chemical contrast (bright positive, dark negative) cannot be securely predicted since various influences exist. We substantiate these facts with a series of typical images.

Shear-force AFM at very rich and high topography of a chemically uniform crystal surface gives no optical contrast in SNOM despite proper reflectance enhancement (Fig. 11). There are no topographical artifacts [2] (further examples in [1]). Chemical contrast is impossible on chemically uniform materials. On the other hand, the bright contrast in the optical image (b) of Fig. (12) reveals positive optical contrast at the chemically different hill site. Fig. (13) gives an example for negative optical contrast at the hill sites.
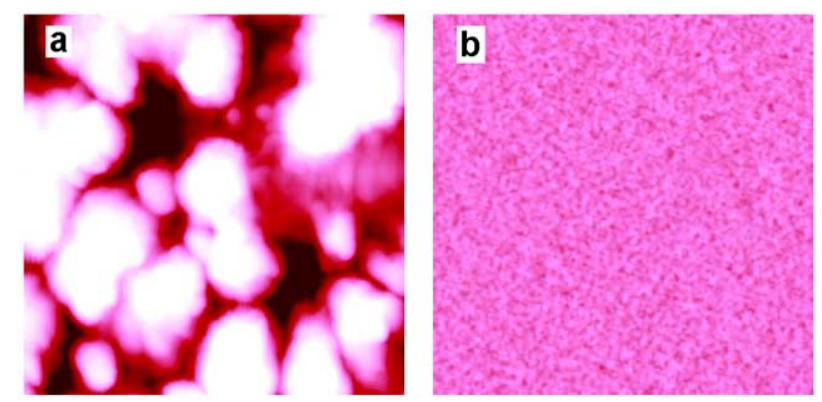

Fig. (11). Simultaneous shear-force AFM with rich topography (a) (10 $\mu \mathrm{m}$, z-range: $500 \mathrm{~nm}$ ) and contrast-less SNOM (b) on a uniform 4-nitroaniline surface with a sharp tip at enhanced reflectance, excluding topographical artifacts [2]. a

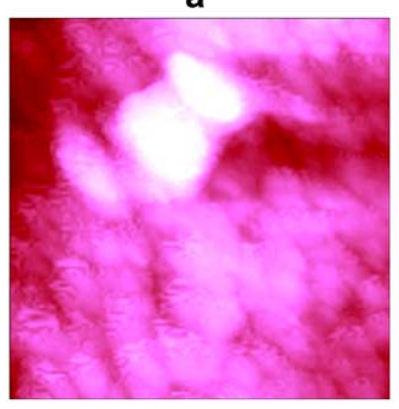

b

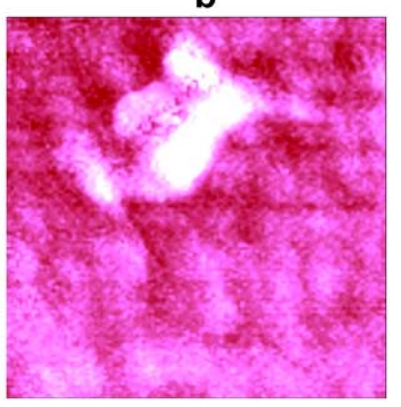

Fig. (12). Structure details within a petrified fossil bacterium on a dolomite sample; (a) shear-force AFM (5 $\mu \mathrm{m}$, z-range: $300 \mathrm{~nm}$ ); (b) simultaneous apertureless SNOM image due to chemical difference with bright contrast at the hill site [35].
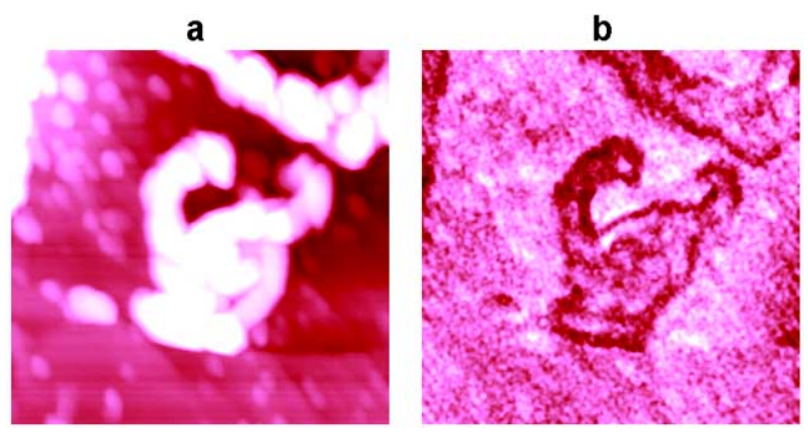

Fig. (13). Shear-force AFM (a) $(11 \mu \mathrm{m}$, z-range: $500 \mathrm{~nm})$ and SNOM (b) of a partially autoxidized (001)-face of 2-mercaptobenzothiazole forming less polar disulfide islands with dark contrast precisely at the island sites [1,23].

Similarly, dark and bright chemical contrasts exist in depressions. The review [1] contains numerous further examples for positive and negative contrasts at well-defined sites, including flat sites, which shows that topographical change is not essential for the optical contrast. Fig. (14) features different optical contrast at nanoparticles. AFM cannot decide if the larger particles of the silver sol (nominal size $200 \mathrm{~nm}$ ) are aggregates or impurity, but SNOM immediately proves impurity by dark contrast. Furthermore, the small particles are not uniform, some exhibiting bright, 
others medium optical contrast within the same image. Again this proves that our SNOM images are free of topographical artifacts, and that the optical contrast is precisely at the same position as the object.
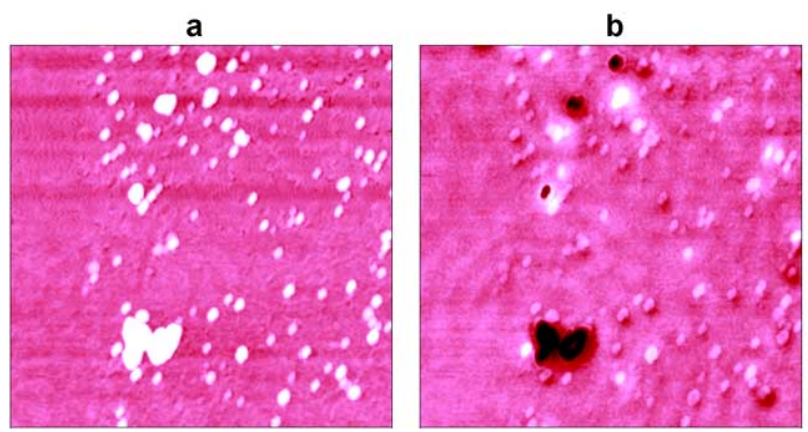

Fig. (14). Shear-force AFM (a) $(10 \mu \mathrm{m}$, z-range: $200 \mathrm{~nm})$ and apertureless SNOM (b) of dried silver sol on a microscope coverslip, with different chemical contrast in the SNOM image (b) [1, 2].

Different contrast in the same image either on heights or depressions is also observed at more complicated topographies of multi-component surfaces. The analysis of a glazed paper with various finely divided minerals using sharp tip and proper reflection enhancement in Fig. (15) reveals bright and dark contrast in the same image on hills, slopes, and depressions. This is again due to different chemical composition at these positions but not to topopraphy.
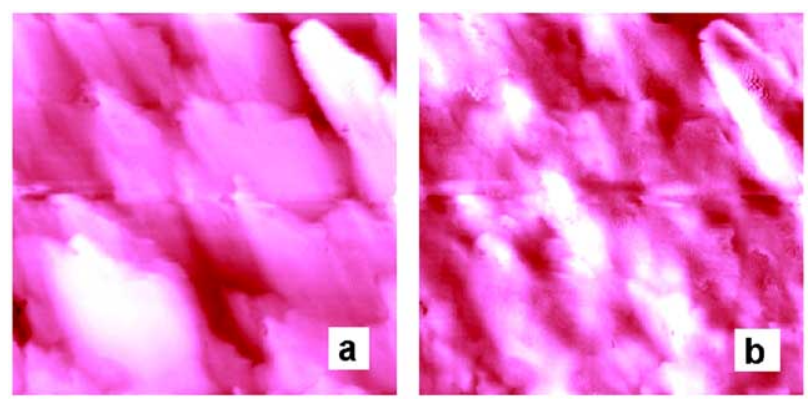

Fig. (15). Shear-force AFM of a glazed paper with roughness (a) (5 $\mu \mathrm{m}$; z-range: $200 \mathrm{~nm}$ ), and apertureless SNOM (b) with bright, medium, and dark contrast at varied positions $[1,2]$.

\section{LOCAL ENHANCED SPECTROSCOPY IN THE SHEAR-FORCE GAP}

The identification of the particles at the various sites for example in Figs. $(\mathbf{1 4}, \mathbf{1 5})$ can be obtained from local Raman SNOM, due to the strict site correspondence. This is exemplified for a further example in Fig. (16). The striking optical contrast in the simultaneous SNOM (b) cannot be expected at all from the AFM image of the natural dolomite surface. A geode must be slightly under the almost flat surface (further images and examples in [8]). The enhanced local Raman SNOM spectrum from within the area of the bright contrast characterized the geode. Fig. (16c) exhibits Raman lines that correspond to those of pyrite $\left(\mathrm{FeS}_{2}\right)$. The resonant local Raman experiment with a $20 \mathrm{~mW}$ Ar-laser

used an optoacoustic fiber spectrometer and edge and notch filters to eliminate the primary light. It required only 145 scanlines. The importance of having precise site correspondence cannot be overemphasized.

$\mathbf{a}$

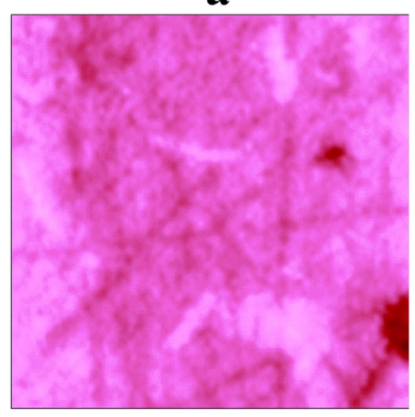

c

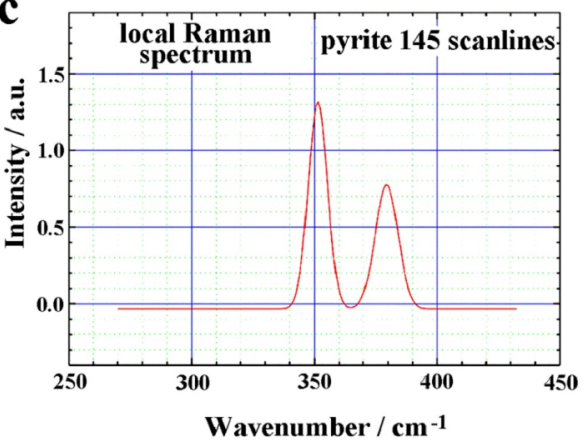

Fig. (16). Shear-force AFM (a) (5 $\mu \mathrm{m}$, z-range: $100 \mathrm{~nm})$ and strong sharp chemical contrast in the SNOM image (b) of a pyrite geode under the dolomite surface, which is not seen in the AFM; (c): enhanced local Raman SNOM spectrum of the pyrite geode only at the bright location in (b); the spectrum is flattened and a baseline subtraction was executed [35].

Similarly, local Raman SNOM spectra of silicon (519.7 $\left.\mathrm{cm}^{-1}\right)$ and its freshly grown $(5 \mathrm{~nm})$ silica layer $\left(500 \mathrm{~cm}^{-1}\right)$ (non-resonant) as well as of gallium nitride on alumina $\left[\mathrm{E}_{1}(\mathrm{TO})\right.$ and $\mathrm{E}_{2}$ Raman modes at 560.8 and $570.4 \mathrm{~cm}^{-1}$ ] at total collection times of $10 \mathrm{~min}$ are reported in [33]. These enhanced Raman emissions on flat and rough surfaces are unsurpassed. The much more involved scattering tipenhanced Raman techniques [20, 21] cannot scan even moderately corrugated surfaces. Also the limitations of aperture Raman SNOM are severe, due to hot blunt tips and long far-field collection times [36-38]. Metal-coated tips feature poor lateral resolution and have difficulties with topography. Surface mapping does not help much.

Double enhancement is reached with apertureless shearforce gap SERS SNOM. For example, the spectrum of a dried solution of adenine $\left(3 \times 10^{-18} \gamma \mathrm{nm}^{-2}\right)$ in the presence of silver particles reproduced the known Raman line at $735 \mathrm{~cm}^{-1}$ in 10 min (Fig. 17). There was only a minute amount of adenine under the tip apex. The total enhancement and sensitivity is excellent. It is only obtainable with emission back-to-the-uncoated-fiber SNOM due to the $200 \mathrm{~nm}$ heights of the silver particles. Numerous local applications also with silver particles on biological samples are envisioned at low collection times [35]. 


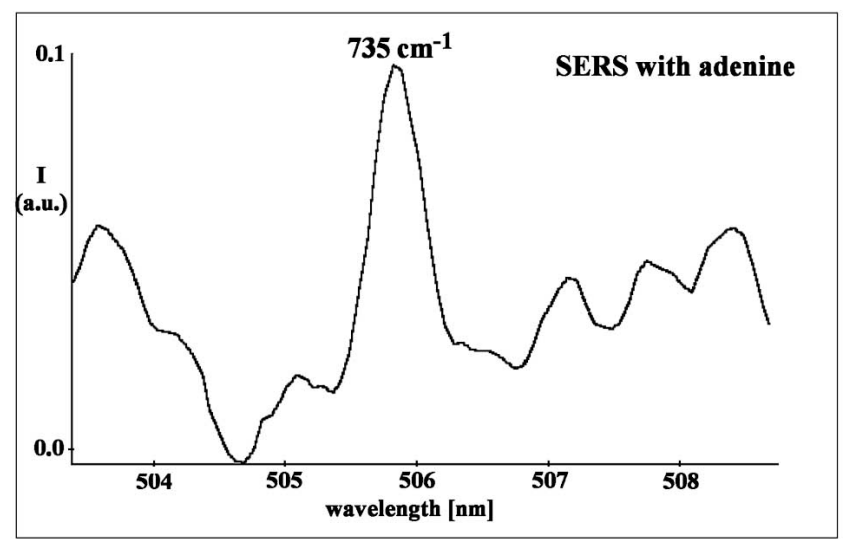

Fig. (17). SERS SNOM spectrum from a $100 \times 100 \mathrm{~nm}^{2}$ area of adenine in the presence of $\mathrm{Ag}$ particles in a dried drop on a microscope cover-slip; raw data without flattening or baseline subtraction [35].

Different other techniques are less efficient and have their problems with topography. Reports are available on apertured tips and silver coated beads [39], gold or silver coated AFM tips as scatterers for a focused laser beam [40$42]$, and metal tips in various configurations [43, 44]. These did not always have the SERS at the local position where it was emitted.

Local fluorescence SNOM by enhanced emission is easier than Raman SNOM. It is highly resolved in the shearforce gap with sharp uncoated tips at better resolution than the AFM [33]. An edge filter eliminates the primary light. Assessment of a $5 \mathrm{~nm}$ varnish cover by comparing fluorescence spots with AFM widths is mentioned in the optical resolution section. Fig. (18, with only one single particle) with precise local coincidence concentrates on the aggregation type. Only local fluorescence SNOM reveals the archly aggregation (further images are in $[1,2]$ ). The resin cover fills the void for closing the topographical object [33].

Another exciting application of enhanced fluorescence SNOM (it does not appear unless the sharp tip is in shearforce distance) is the determination of diffusion coefficients $(D)$ from the intensity drops in ring-dyed textile fibers, by applying Fick's second law of diffusion and dyeing details (Fig. 19). The resulting $D$-values were found constant over the complete depletion curve. The absolute value of the diffusion coefficient (dyeing time $30 \mathrm{~min}$ ) is calculated to $D_{130^{\circ} \mathrm{C}}=2.4 \times 10^{-11} \mathrm{~cm}^{2} \mathrm{~s}^{-1}$. This value is consistent with similar values in the literature. Further examples also for protruding fibers are in $[1,33]$. Such measurements are apparently out of reach for conventional SNOM with dyed hair. No diffusion coefficients could be determined with transmission images using a long-pass filter at very low scan rates with a claimed optical resolution of $130 \mathrm{~nm}$ [45].

Virtually all SNOM modes tried fluorescence imaging despite topographical artifacts and often non-coincidence of fluorescence with fluorescing object location and poor resolution. Two-photon near-field fluorescence microscopy has also been reported [46]. Furthermore, second harmonics generation [47] at the metal tip as well as a white continuum has been observed [48]. These techniques require pico- and femto-second laser pulses focused to the metal tip on the surface. A drawback of these techniques is photobleaching that also occurs with nanofluorescence derived "active tips" [2] and the more recent STED technique.
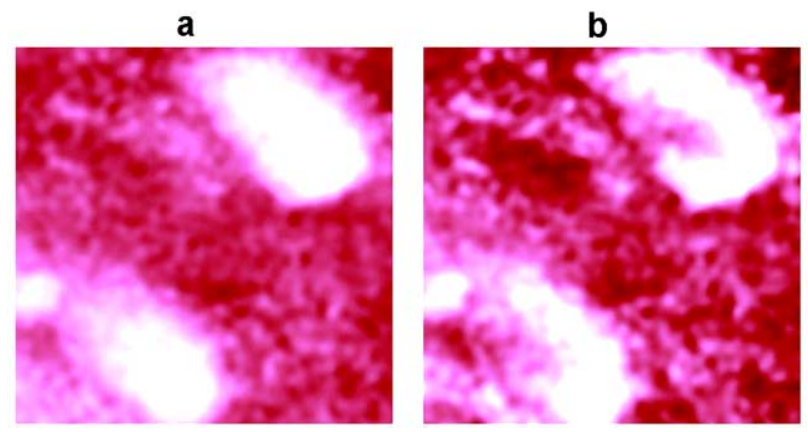

Fig. (18). Simultaneous shear-force AFM $(1.2 \mu \mathrm{m}$; z-scale $200 \mathrm{~nm})$ (a) and enhanced fluorescence SNOM (b) of dye nanoparticles in polyvidone resin revealing archly aggregation; primary wavelength was $488 \mathrm{~nm}$ [2]. a
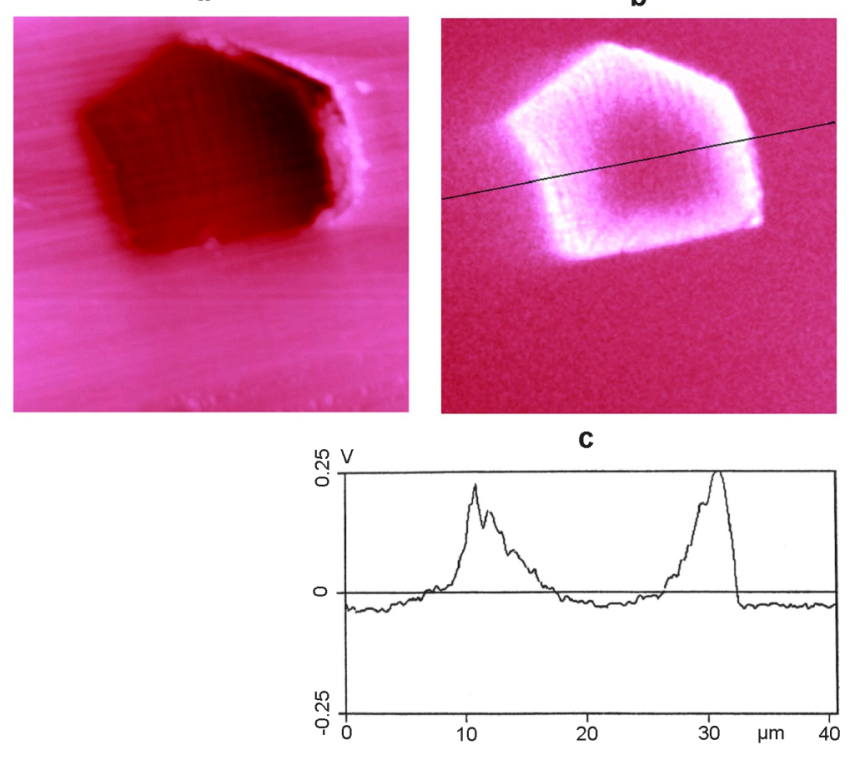

b

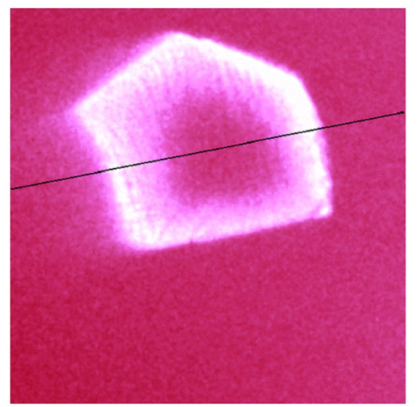

C
Fig. (19). Simultaneous shear-force AFM (a) $\left(40 \mu \mathrm{m}, 200 \mu \mathrm{m} \mathrm{s}^{-1}\right.$, z-range: $2 \mu \mathrm{m}$ ) and enhanced fluorescence SNOM (b) of a technical fluorescing dye in a ring-dyed polyester fiber, as embedded in Technovit 7100 resin for a microtome cut; (c): cross section through fluorescence, as indicated in (b); edge filter OG 515 (Schott, Germany); steeper right decay curve taken for determination of $D[1,33]$.

\section{ARTIFACTS}

A useful topographical artifact at a vertical slope of a cubic $\mathrm{NaCl}$ crystal is mentioned in the optical resolution section. There are also situations with overhangs that must enforce the artificial optical stripes contrast around the true chemical contrast for example in the aggregated latices in Fig. (20) [49]. They are dark and brighter at their dipolar contact sites and these attract a water layer. The stripes artifact top-to-bottom is resolved at $<20 \mathrm{~nm}$ by analysis of the cross section (c). These beads are not elongated in closed monolayers. They have important parallels in biological objects [35]. These results again underline the superiority of reflection back-to-the-uncoated-fiber SNOM, the easy and versatile technique. 


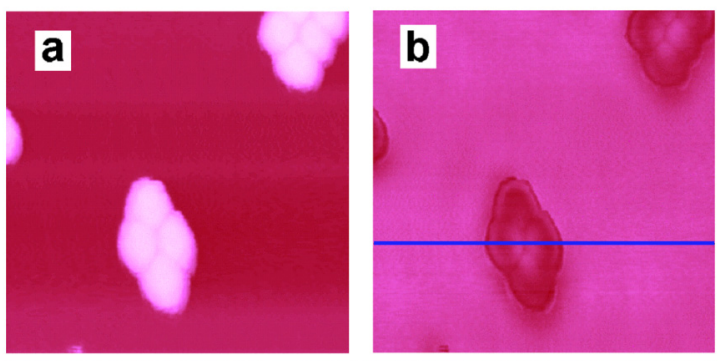

c

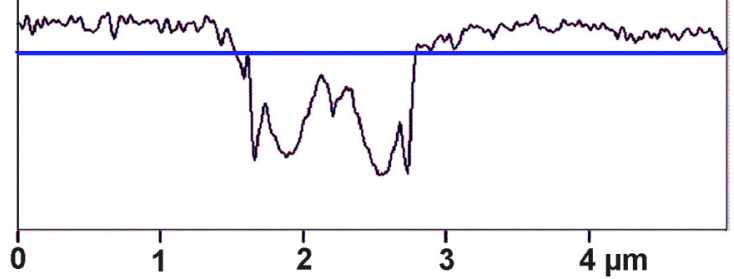

Fig. (20). Shear-force AFM (a), apertureless SNOM (b) with cross section (c) of small aggregates of $430 \mathrm{~nm}$ styrene-acrylamide copolymer latices on mica showing both stripes contrast and chemical contrast.

The SNOM literature is full of artifacts (non-chemical contrast). These are similar in all techniques of Fig. (1). Useless artifacts in our apertureless SNOM occur only if broken tips replaced sharp ones with exclusion of the reflectance enhancement. Various situations occur depending on the tip shape after breakage. All artifact types are comprehensively summarized and imaged in [50] for enabling immediate judgment of reported claims of SNOM contrast (e.g. artificial $1 \mathrm{~nm}$ resolution claim in [19]). The artifacts appear as contrast reversal, inverted contrast, interference fringes, stripes contrast, inverted derivative of the topography in the optical channel, displaced optical contrast, far-field light concentration, and topographical artifacts (e.g. "contrast" from chemically uniform surface).

\section{CONCLUSIONS}

Proper SNOM on rough surfaces must be robust, versatile, and economic. Reflection back-to-the-fiber in the shear-force gap SNOM meets these criteria with near-field collection. Its reported basics substantiate the principal differences of this apertureless SNOM with respect to all other SNOM techniques. It depends on the apparently unexpected strong enhancement of the reflected light intensity. The easily followed experimental practice and control guarantees proper conditions that must be obeyed to avoid artifacts with blunt or broken tips. The enhancement makes it independent from topography, as long as the horizontally vibrating sharp tip with high aspect ratio can follow the positive and negative slopes (of course stripes artifact occurs at verticals and overhangs). This also implies that the optical contrast is found exactly at the position where the chemical difference resides and this is also a necessary requirement for interpretable local spectroscopy. True SNOM contrast differentiates chemical entities und is therefore chemical contrast. All of that has been undoubtedly demonstrated with a selection of typical simultaneous AFM and SNOM images for all thinkable situations. Also implied is the same (or better) optical resolution when compared to AFM resolution. It is shown to be $<10 \mathrm{~nm}$ in favorable or $<$ $20 \mathrm{~nm}$ in topographical situations. Only the pixel size $(512 \mathrm{x}$ 512 pixels) limits the resolution in large scans. There is an important difference to conventional aperture SNOM (including near-field IR, thermal, and microwave techniques): the site correspondence is not seen and not possible with metal-coated tips that cannot reasonably follow any roughness of a surface without continuous changes of the distance between aperture and sample surface, because the ever changing closest edge point of the coating to any slope is responsible for the distance control. Furthermore, about $100 \mathrm{~nm}$ wide apertures cannot reach at all the excellent optical resolution of the uncoated tips in our easy and versatile technique for rough and natural surfaces with the already reviewed applications in virtually all fields of science and technique including medicine and relevance to daily life aspects (for example withdrawal of a widely used dental alloy from the market on the basis of chemical contrast measurements, (pre)cancer diagnosis, biocompatibility, etc) [1]. The present SNOM technique relies on the specific response of the materials surface to near-field light, but not on geometrical effects. The versatile results and applications are not available by any other technique. The apertureless shear-force technique is far less complicated than more recent apertureless SNOM modes with laser focusing to a near-field probe with far-field detection and modulation requirements for extraction of the optical image, techniques that cannot scan on rough surfaces.

\section{REFERENCES}

[1] Kaupp G. Scanning near-field optical microscope on rough surfaces: applications in chemistry, biology, and medicine. Int $\mathrm{J}$ Photoener 2006; ID 69878: 1-22.

[2] Kaupp G. Atomic force microscopy, scanning nearfield optical microscopy and nanoscratching: application to rough and natural surfaces - springer series nanoscience and technology. Springer, Heidelberg 2006; Erratum at: ISBN 978-3-540-28405-5.

[3] Cricenti A. Scanning near-field optical microscopy (SNOM). Phys Stat Sol 2008; 5: 2615-20.

[4] Antosiewicz TJ, Wrobel P, Szoplik T. High resolution SNOM probes. Proc SPIE 2008; 7141: 71411I/1-/8.

[5] Zenobi R. New methods for molecular analysis on the nanometer scale. Chim l'Ind 2008; 90: 92-7.

[6] De Wilde Y, Lemoine PA. Review of NSOM microscopy for materials. AIP Conf Proc 2007; 931: 43-52.

[7] Sandoghdar V, Wegscheider S, Krausch G, Mlynek J. Reflection scanning near-field microscopy with uncoated fiber tips: How good is the resolution really? J Appl Phys 1997; 81: 2499-503.

[8] Kaupp G, Herrmann A, Schmeyers J, Boy J. Optical near-field microscopy and -spectroscopy. Part: optical near-field microscopy on rough surfaces using tapered waveguide tips in cross polarization. Ref. No. 13N7640/3: 1-24; Final report. Bundesministerium für Bildung und Forschung (BMBF): Bonn, Germany. Available from: Technische Informations-Bibliothek TIB, POBox 8080, D-30060 Hannover, Germany 2003.

[9] Shalaev VM, Cai W, Chettiar UK, et al. Negative index of refraction in optical metamaterials. Opt Lett 2005; 30: 3356-8.

[10] Wrobel P, Pniewski J, Antosiewicz TJ, Szoplik T. Focusing of radially polarized light with corrugated silver nanolayer. Proc SPIE 2009; 7353: 73530X-X-9.

[11] Dyba M, Hell SW. Focal spots of size lambda/23 open up far-field fluorescence microscopy at $33 \mathrm{~nm}$ axial resolution. Phys Rev Lett 2002; 88: 163901-1/-4.

[12] Zumbusch A. High-resolution light microscopy. Nachr Chem 2009; 57: 294-6.

[13] Cusano A, Pilla P, Consales M, et al. Near field behavior of $\mathrm{SnO}_{2}$ particle-layer deposited on standard optical fiber by electrostatic spray pyrolysis method. Opt Express 2007; 15; 5136-46. 
[14] Buosciolo A, Consales M, Pisco M, Cusano A, Giordano M. Fiberoptic near-field chemical sensors based on wavelength scale tin dioxide particle layers. J Lightwave Technol 2008; 26: 3468-75.

[15] Lei F. Manfait M. Non-optical bimorph-based force sensor for scanning near-field optical microscopy of biological materials: characteristics, design and applications. Surf Interface Anal 2007; 39: 674-83.

[16] Reddick RC, Warmack RJ, Ferrel TL. New form of scanning optical microscopy. Phys Rev B 1989; 39: 767-70.

[17] Aubert S, Bruyant, S. Blaize, R, et al. Analysis of the interferometric effect of the background light in apertureless scanning near-field optical microscopy. J Opt Soc Am B 2003; 20: 2117-24.

[18] Brückl H, Pagnia H, Sotnik N. Optical near-field characterization of submicron structured silicon films. Scanning 1995; 17: 24-7.

[19] Zenhausen F, O'Boyle MP, Wickramasinghe HK. Apertureless near-field optical microscope. Appl Phys Lett 1994; 65: 1623-5.

[20] Keilmann F, Hillenbrand R. Near-field microscopy by elastic light scattering from a tip. Phil Trans Roy Soc London, Ser A 2004; 362 : 787-805.

[21] Patane S, Gucciardi PG, Labardi M, Allegroni M. Apertureless near-field optical microscopy. Riv Nuovo Cimento Soc Ital Fis 2004; 27: 1-46.

[22] Kaupp G, Herrmann A, Haak M. Near-field optical microscopy with uncoated tips: calibration, chemical contrast on organic crystals, and photolithography. J Vac Sci Technol 1997; B 15: $1521-6$.

[23] Kaupp G, Herrmann A. Chemical contrast in scanning near-field optical microscopy. J Phys Org Chem 1997; 10: 675-9.

[24] Kaupp G, Herrmann A, Schmeyers J, Boy J. SNOM: a new photophysical tool. J Photochem Photobiol A: Chem 2001; 139: 93-6.

[25] Madsen S, Bozhevolnyi SI, Hvam JM. Sub-wavelength imaging by depolarization in a reflection near-field optical microscope using an uncoated fiber probe. Opt Commun 1998; 146: 277-84.

[26] Grober RD, Harris TD, Trautman JK, Betzig E. Design and implementation of a low temperature near-field scanning optical microscope. Rev Sci Instrum 1064; 65: 626-31.

[27] Schüttler M, Leuschner M, Lippitz M, Rohle W, Giessen H. Towards the origin of the shear force in near-field microscopy. Jpn J Appl Phys 2001; 40: 813-8.

[28] Courjon D, Vigoureux JM, Spajer M, Sarayeddine K, Leblanc S. External and internal reflection near field microscopy: experiments and results. Appl Opt 1990; 29: 3734-40.

[29] Kaupp G. Supermicroscopy in supramolecular chemistry: AFM, SNOM, and SXM. In: Davies JED, Ripmeester JA, Eds. Comprehensive supramolecular chemistry. Elsevier, Oxford: 1996; vol. 8: pp. 381-423.

[30] Kaupp G, Herrmann A. Scanning near-field optical microscopy by near-field reflectance enhancement: a versatile and valid technique. J Phys Org Chem 1999; 11: 141-3.

[31] Adelmann C, Hetzler J, Scheiber G, et al. Experiments on the depolarization near-field scanning optical microscope. Appl Phys Lett 1999; 74: 179-81.

[32] Zhang T, Fang Z, Zheng J, et al. The influence of humidity on the shear force between tip and sample in NSOM using piezoelectric fork. Surf Rev Lett 2005; 12: 355-8.
[33] Kaupp G, Herrmann A, Wagenblast G. Scanning near-field optical microscopy (SNOM) with uncoated tips. Proc SPIE 1999; 3607: 16-25.

[34] Madsen S, Bozhevolnyi SI, Hvam JM. Sub-wavelength imaging by depolarization in a reflection near-field optical microscope using an uncoated fiber probe. Opt Commun 1998; 146: 277-84.

[35] Kaupp G, Herrmann A, Naimi-Jamal MR, Stepanenko S, Stepanenko V. Laseroptic procedures for differentiated characterization of bio systems. Part: Near-field microscopy for organic and biological surfaces. Ref. No. 13N7519: 1-33; Final report. Bundesministerium für Bildung und Forschung (BMBF) Bonn, Germany. Available from: Technische Informationsbibliothek TIB, POBox 8080, D-30060 Hannover, Germany 2003.

[36] Zavalin A, Cricenti A, Generosi R, Luce M, Morgan S, Piston D. Collection mode nano-Raman setup. Phys Stat Sol C 2005; 2: 4106-10.

[37] Smith DA, Webster S, Ayad M, Evans SD, Fogherty D, Batchelder D. Development of a scanning near-field optical probe for localized Raman spectroscopy. Ultramicroscopy 1995; 61: 247-52.

[38] Webster S, Batchelder DN, Smith DA. Submicron resolution measurement of stress in silicon by near-field Raman spectroscopy. Appl Phys Lett 1998; 72: 1478-80.

[39] Deckert V, Zeisel D, Zenobi R. Near-field surface-enhanced Raman imaging of dye-labeled DNA with 100-nm resolution. Anal Chem 1998; 70: 2646-50.

[40] Anderson MS. Locally enhanced Raman spectroscopy with an atomic force microscope. Appl Phys Lett 2000; 76: 3130-2.

[41] Hayazawa N, Inouye Y, Sekkat Z, Kawata S. Near-field Raman imaging of organic molecules by an apertureless metallic probe scanning optical microscope. J Chem Phys 2002; 117: 1296-301.

[42] Hartschuh A, Sanchez EJ, Xie XS, Novotny L. High-resolution near-field Raman microscopy of single-walled carbon nanotubes. Phys Rev Lett 2003; 90: 095503-1/-4.

[43] Stöckle RM, Suh YD, Deckert V, Zenobi R. Nanoscale chemical analysis by tip-enhanced Raman spectroscopy. Chem Phys Lett 2000; 318: 131-6.

[44] Stöckle RM, Deckert V, Fokas C, Zeisel D, Zenobi R. Subwavelength Raman spectroscopy on isolated silver islands. Vibr Spectrosc 2000; 22: 39-48.

[45] Kelch A, Wessel S, Will T, Hintze U, Werpf R, Wiesendanger R. Penetration pathways of fluorescent dyes in human hair fibres investigated by scanning near-field optical microscopy. J Microsc 2001; 200: 179-86.

[46] Sanchez EJ, Novotny L, Xie XS. Near-field fluorescence microscopy based on two-photon excitation with metal tips. Phys Rev Lett 1999; 82: 4014-7.

[47] Labardi M, Allegrini M, Zavelani-Rossi M, et al. Highly efficient second-harmonic nanosource for near-field optics and microscopy. Opt Lett 2004; 29: 62-4.

[48] Bouhelier A, Beversluis MR, Novotny L. Application of fieldenhanced near-field optical microscopy. Ultramicroscopy 2004; 100: 413-9.

[49] Teixeira-Neto E, Kaupp G, Galembeck F. Latex particle heterogeneity and clustering films. J Phys Chem B 2003; 107: 14255-60.

[50] Kaupp G, Herrmann A, Haak M. Artifacts in topography and scanning near-field optical microscopy (SNOM) due to deficient tips. J Phys Org Chem 1999; 12: 797-807. 